\title{
Tuning the Electronic Structure of Metal-Oxygen Group by Silicon Substitution in Lithium-Rich Manganese-Based Oxides for Superior Performance
}

Jin, Ma; Yan, Huijun; Li, Biao; Xia, Zhonghong; Huang, Weifeng; An, Li; Xia, Dingguo *
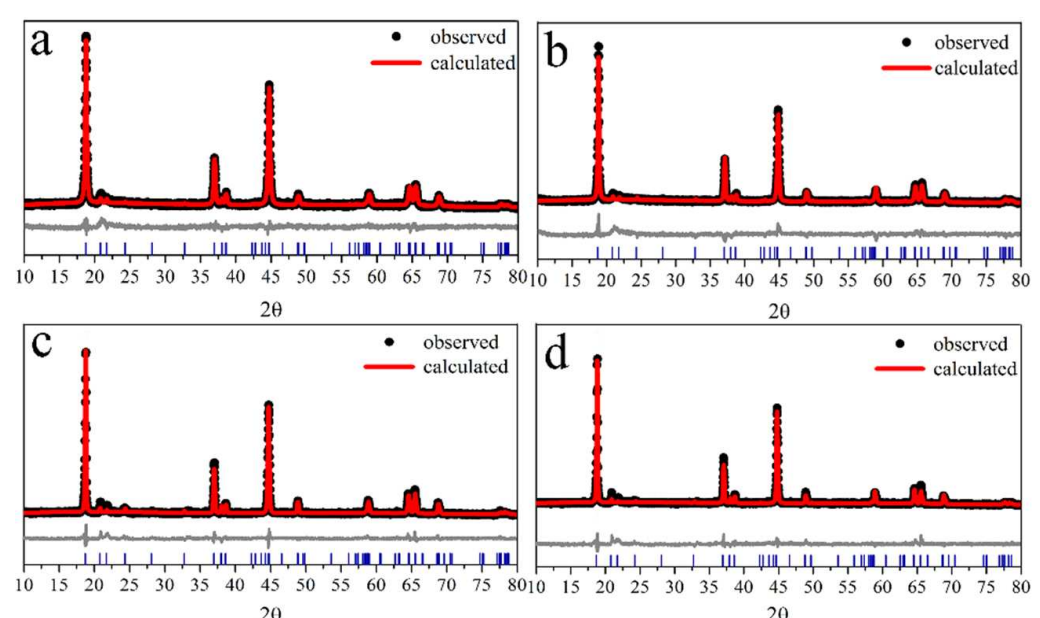

Fig. S1 fitting results of LNCMO, LNCMO-Si0.02, LNCMO-Si0.04 and LNCMO-Si0.06

a. $\mathrm{LNCMO}, \mathrm{R}_{\mathrm{exp}}=5.79, \mathrm{R}_{\mathrm{wp}}=7.35, \mathrm{R}_{\mathrm{p}}=5.64, \mathrm{GOF}=1.27$

b. $\mathrm{LNCMO}-\mathrm{Si} 0.02, \mathrm{R}_{\mathrm{exp}}=5.45, \mathrm{R}_{\mathrm{wp}}=7.62, \mathrm{R}_{\mathrm{p}}=5.62, \quad \mathrm{GOF}=1.40$

c. $\mathrm{LNCMO}-\mathrm{Si0} .04, \mathrm{R}_{\exp }=5.46, \mathrm{R}_{\mathrm{wp}}=8.36, \mathrm{R}_{\mathrm{p}}=6.26, \mathrm{GOF}=1.53$

d. LNCMO-Si0.06, $\mathrm{R}_{\exp }=6.34, \mathrm{R}_{\mathrm{wp}}=9.17, \mathrm{R}_{\mathrm{p}}=7.09, \mathrm{GOF}=1.45$

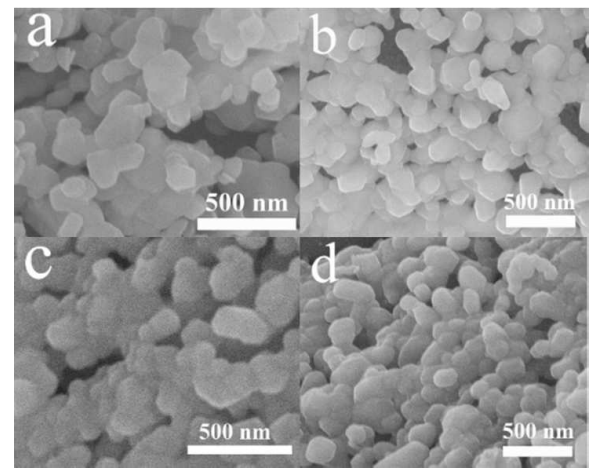

Fig. S2 SEM images of (a) LNCMO, (b) LNCMO-Si0.02 (c) LNCMO-Si0.04 (d) LNCMO-Si0.06 


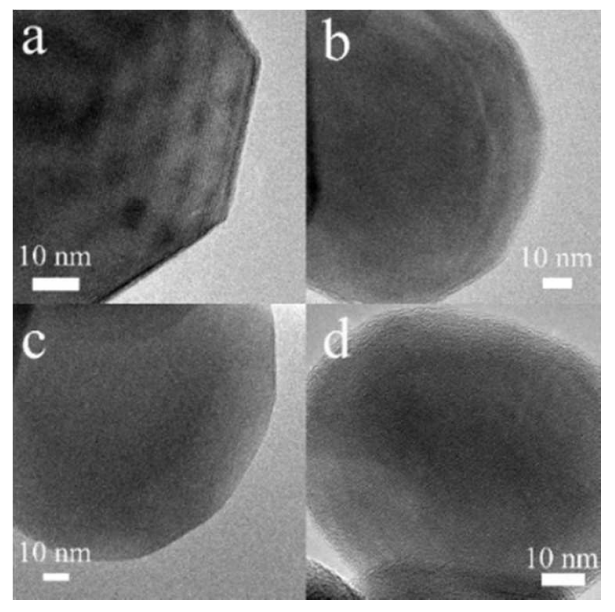

Fig. S3 TEM images of (a) LNCMO, (b) LNCMO-Si0.02 (c) LNCMO-Si0.04 (d) LNCMO-Si0.06
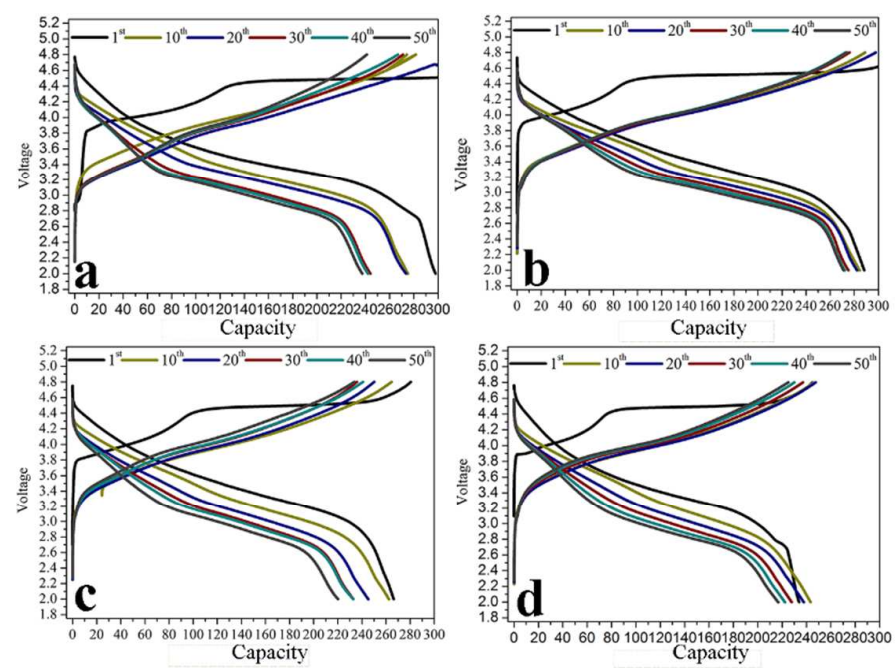

Fig. S4 Voltage evolution of (a) LNCMO, (b) LNCMO-Si0.02, (c) LNCMO-Si0.04 , (d) LNCMO-Si0.06. 

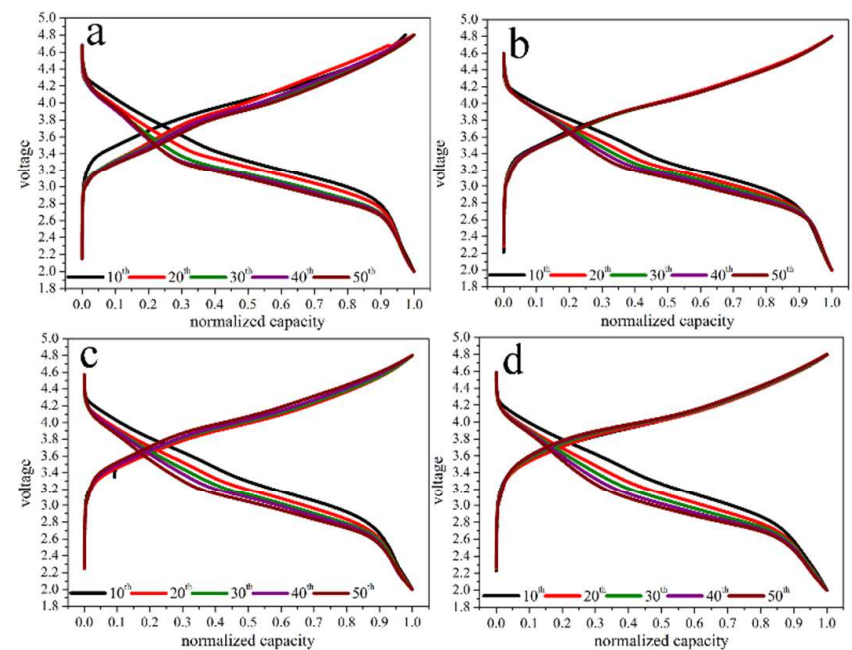

Fig. S5 Voltage evolution of (a) LNCMO, (b) LNCMO-Si0.02, (c) LNCMO-Si0.04 , (d)

LNCMO-Si0.06 with normalized discharge capacity.

Table S1 lattice parameters acquired by XRD fitting

\begin{tabular}{cccccc}
\hline \multirow{2}{*}{ sample } & \multicolumn{5}{c}{ lattice parameter } \\
\cline { 2 - 6 } & $\mathrm{a}(\AA)$ & $\mathrm{b}(\AA \AA)$ & $\mathrm{c}(\AA)$ & $\beta\left({ }^{\circ}\right)$ & $\mathrm{V}\left(\AA^{3}\right)$ \\
\hline LNCMO & $4.93598(43)$ & $8.55079(52)$ & $5.01120(68)$ & $109.047(12)$ & $199.926(37)$ \\
LNCMO-Si0.02 & $4.92838(40)$ & $8.53789(36)$ & $5.01302(46)$ & $109.0137(78)$ & $199.429(27)$ \\
LNCMO-Si0.04 & $4.92871(31)$ & $8.53757(36)$ & $5.01218(47)$ & $109.0126(74)$ & $199.403(26)$ \\
LNCMO-Si0.06 & $4.92868(65)$ & $8.53661(98)$ & $5.01146(40)$ & $109.0030(75)$ & $199.362(39)$ \\
\hline
\end{tabular}

Table S2 the component analysis of LNCMO, LNCMO-Si0.02, LNCMO-Si0.04 and LNCMO-Si0.06 materials by Inductive coupled plasma atomic emission spectrometer (ICP-AES)

\begin{tabular}{ccccc}
\hline Sample & element & $\begin{array}{c}\text { Mean } \\
(\mathrm{ug} / \mathrm{mL})\end{array}$ & $\begin{array}{c}\text { RSD } \\
(\mathrm{ug} / \mathrm{mL})\end{array}$ & $\begin{array}{c}\text { atomic } \\
\text { ratio }\end{array}$ \\
\hline \multirow{2}{*}{ LNCM0 } & Li & 8.8006 & 0.1679 & 1.26 \\
& Mn & 31.6309 & 0.188 & 0.57 \\
& Co & 7.8964 & 0.1946 & 0.13 \\
& $\mathrm{Ni}$ & 7.895 & 0.2243 & 0.13 \\
\hline LNCM0-S & Li & 7.6752 & 0.2653 & 1.10 \\
i0.02 & Co & 7.4881 & 0.2975 & 0.12 \\
& $\mathrm{Ni}$ & 8.1171 & 0.4941 & 0.13 \\
& $\mathrm{Si}$ & 0.6731 & 0.2669 & 0.02 \\
\hline LNCM0-S & Li & 9.4123 & 0.2931 & 1.35 \\
i0.04 & Mn & 31.1939 & 0.2976 & 0.56 \\
\hline
\end{tabular}




\begin{tabular}{ccccc} 
& Co & 8.2492 & 0.1898 & 0.13 \\
& $\mathrm{Ni}$ & 7.5949 & 0.1494 & 0.13 \\
& $\mathrm{Si}$ & 1.3595 & 0.1266 & 0.04 \\
\hline & $\mathrm{Li}$ & 8.4164 & 0.1681 & 1.21 \\
LNCM0-S & $\mathrm{Mn}$ & 28.6477 & 0.2157 & 0.52 \\
i0. 06 & Co & 8.8517 & 0.218 & 0.15 \\
& $\mathrm{Ni}$ & 10.0866 & 0.3734 & 0.17 \\
& $\mathrm{Si}$ & 1.9031 & 0.1211 & 0.06 \\
\hline
\end{tabular}

Table S3 simulated impedance parameters from equivalent circuit

\begin{tabular}{cccc}
\hline & $\mathrm{R}_{1}$ (ohm) & $\mathrm{R}_{2}$ (ohm) & $\mathrm{R}_{3}$ (ohm) \\
\hline LNCM0 & 3.74 & 100.38 & 78.38 \\
LNCMO-Si0.02 & 3.36 & 62.28 & 61.84 \\
LNCMO-Si0.04 & 3.711 & 99.76 & 71.67 \\
LNCMO-Si0.06 & 4.739 & 110.5 & 75.13 \\
\hline
\end{tabular}

